\title{
Epithelioid Glioblastoma with Leptomeningeal Gliomatosis - Case Report and Review of the Literature
}

\author{
Walid Albanna*1,2, Gerrit A Schubert ${ }^{1}$, Hans Clusmann' ${ }^{1}$, Sonny K Tan ${ }^{1}$, Peter Schmiegelow ${ }^{3}$ and Ralf M Buhl ${ }^{2}$ \\ ${ }^{1}$ Department of Neurosurgery, RWTH Aachen University, Germany
}

${ }^{2}$ Department of Neurosurgery, Staedtisches Klinikum Solingen, Germany

${ }^{3}$ Department of Pathology, Staedtisches Klinikum Solingen, Germany

Received: 制: October 10, 2018; Published: 制: October 22, 2018

*Corresponding author: Walid Albanna, Department of Neurosurgery, RWTH Aachen University, Pauwelsstr. 30, 52074 Aachen, Germany

\begin{abstract}
Glioblastomas tend to be morphologically diverse. Although rare, pseudoepithelial components (adenoid or epithelioid) can be a diagnostic challenge. We describe a case of a 47-year-old female patient initially complaining of neck pain and dizziness. On neurological examination, an unsteady gait and memory disturbances were noted. MRI showed a contrast-enhancing lesion in the left temporal lobe without significant edema, suggesting the presence of cerebral metastasis. After complete resection of the tumor (as verified by early post-op MRI), the patient made an uneventful recovery. Based on extensive histopathological analyses, the tumor was diagnosed as epithelioid glioblastoma (E-GBM). Two months after concomitant radiation and chemotherapy, the patient developed severe neck pain, became dizzy and was confused. CSF examination confirmed tumor cells, and MRI of the spine showed diffuse dural contrast enhancement, suggesting extensive leptomeningeal spread. The patient passed away four months after the initial diagnosis. E-GBMs represent one of the least prevalent morphologic subtypes of glioblastoma. Although E-GBMs are difficult to detect and often do not present as high-grade gliomas, clinicians should be aware of the unusual patterns of these GBMs. E-GBM should be considered in the case of MRI findings without peripheral edema, and when newly diagnosed intracerebral masses arise without a known primary. In addition, we discuss our case in terms of previous reports on this condition.
\end{abstract}

Keywords: Adenoid; Epithelioid Glioblastoma; Leptomeningeal; GBM

Abbreviations: E-GBM: Epithelioid Glioblastoma; EMA: Epithelial Membrane Antigen; GFAP: Glial Fibrillary Acidic Protein; MRI: Magnetic Resonance Imaging; CSF: Cerebrospinal Fluid; HE: Hematoxylin and Eosin

\section{Introduction}

Glioblastomas are the highest-grade malignant brain tumors within the spectrum of astrocytic neoplasms [1,2]. A very uncommon morphologic variation is glioblastomas with epithelial or pseudoepithelial (adenoid or epithelioid) features. Epithelioid glioblastomas (E-GBMs) are a rare subtype characterized by a cohesive architectural organization in epithelioid nests and sheets. This phenotype can obscure the glial lineage and may mimic metastatic involvement of non-CNS neoplasms because of epithelial-like characteristics [1,3-5]; thus, E-GBMs remain a considerable diagnostic challenge. Many reports have described these unusual glioblastomas as solitary lesions [6,7]. However, Gasco et al. reported the first case of a multifocal E-GBM that resembled cerebral metastasis [6]. In the present work, we describe an unusual occurrence of an E-GBM with leptomeningeal gliomatosis and discuss it in terms of the available literature.

\section{Clinical Summary}

A 47-year-old right-handed female patient complained of neck pain and dizziness. Neurological examination showed an unsteady gait and memory disturbances with no other focal neurologic deficits. Magnetic resonance imaging (MRI) showed a contrastenhancing lesion in the left temporal lobe without significant surrounding edema, suggesting the presence of a cerebral metastasis (Figure 1). Whole-body MRI screening for a non-CNS primary was negative. Intraoperatively, the tumor was well-defined and demarcated with respect to the surrounding brain tissue, but it extended into the temporal horn, which thus had to be opened during resection. An early post-operative MRI showed complete resection of the contrast-enhancing lesion. The patient recovered quickly from surgery. 

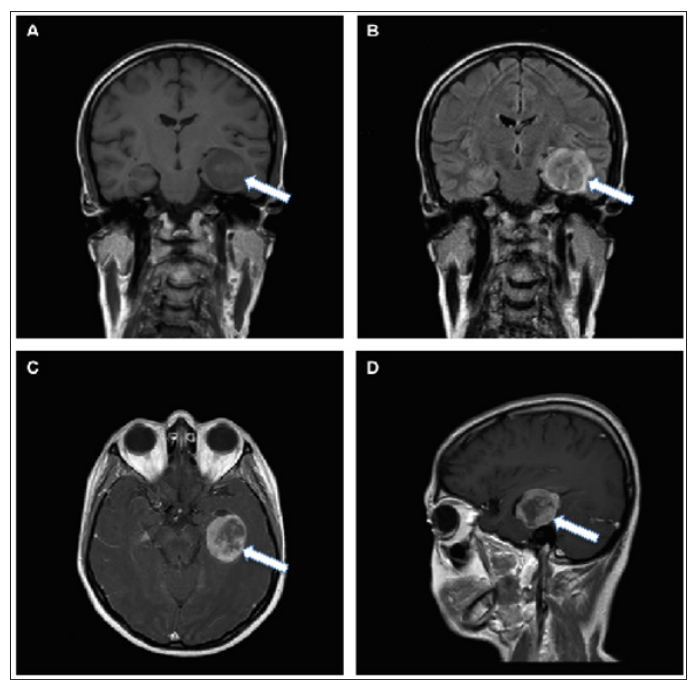

Figure 1: Imaging features of an epithelioid glioblastoma on an MRI scan.

a. Coronal T1-weighted image without gadolinium contrast showing a solid hypointense mass (white arrows) within the temporal lobe;

b. Coronal T2-weighted image showing minimal edema around the tumor;

c. Axial T1-weighted MRI scan with heterogeneous gadolinium enhancement;

d. Sagittal T1-weighted MRI scan with gadolinium implementation displaying a heterogeneously enhancing, round mass.

\section{Pathological Findings}

Extensive histopathological analysis revealed an epithelioid neoplasm comprised of variably sized cells with eosinophilic cytoplasm, round cell morphology, nuclear atypia and mitotic figures (Figure 2). The tumor also showed microvascular proliferation. These morphologic features were compatible with metastatic epithelioid carcinoma. However, additional immunohistochemistry showed positive staining for glial fibrillary acidic protein (GFAP) and epithelial membrane antigen (EMA) (Figure 3); thus, the tumor was diagnosed as an E-GBM. Two months after concomitant radiation and chemotherapy, the patient developed severe neck pain and confusion. MRI of the spine showed diffuse dural contrast enhancement, indicating the presence of extensive leptomeningeal spread (Figure 4). Pathological examination of the cerebrospinal fluid (CSF) confirmed the presence of tumor cells with mitotic activity (Figure 5) and revealed a reproducible low glucose content (Table 1). The decision for palliative treatment was made, and the patient passed away four months after the initial diagnosis.
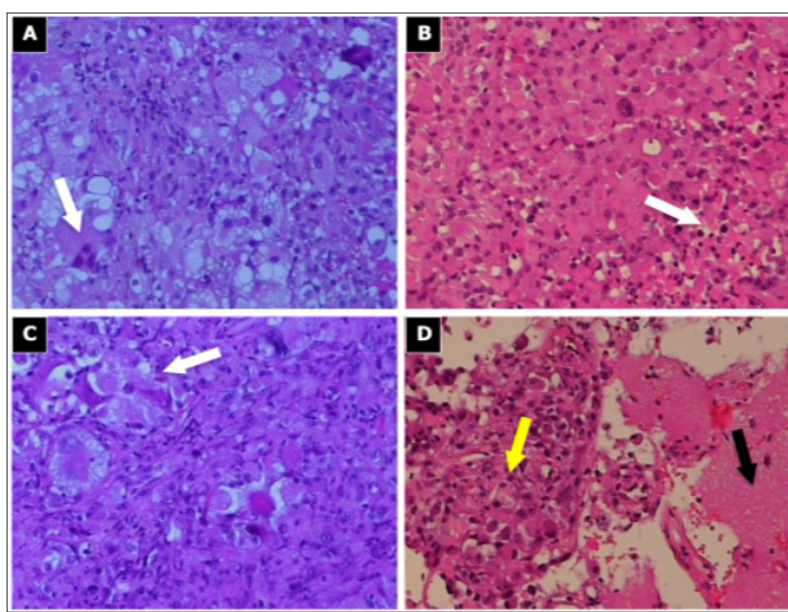

Figure 2: The relevant / classical pathohistological finding of the specimen.

Note: The tissue contains atypical monotonous epithelioid as well as giant atypical multinucleated cells with scant cytoplasm and bipolar fine cell processes;

a. Severe nuclear atypia and rhabdoid cells with scant cytoplasm (hematoxylin and eosin (HE), 200x);

b. Monotonous medium-sized cells with morphologic features of nucleoli and eosinophilic cytoplasm, illustrating classical features of epithelioid glioblastomas (E-GBM), extensive necrosis as indicated in the lower right (white arrow) (HE, 200x);

c. Pronounced atypia of the giant glioblastoma cells (HE, 200x);

d. Transitional tumor zone (yellow arrow) and normal tissue (black arrow) (HE, 100x). 

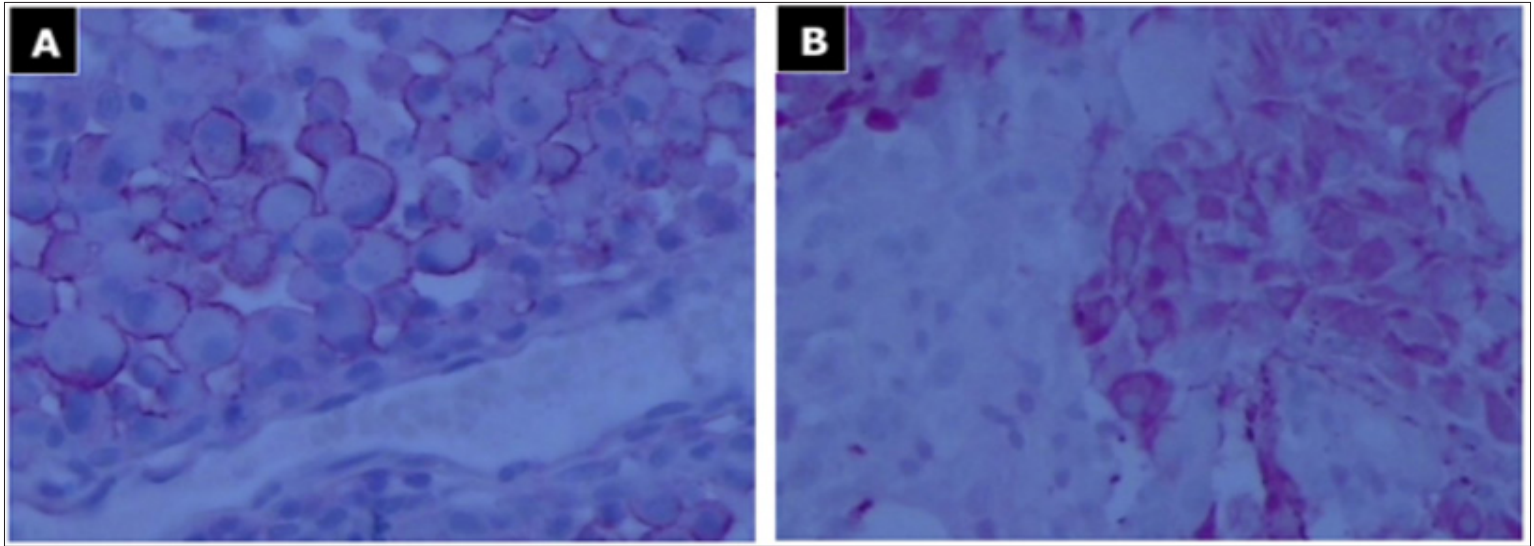

Figure 3: Immunohistochemical expression of E-GBM.

a. Epithelial membrane antigen (EMA) expression in E-GBMs: positive (fast red, 200x);

b. Immunohistochemistry for glial fibrillary acidic protein (GFAP): positive (fast red, 200x). Vimentin and AE1/3 were not determined.
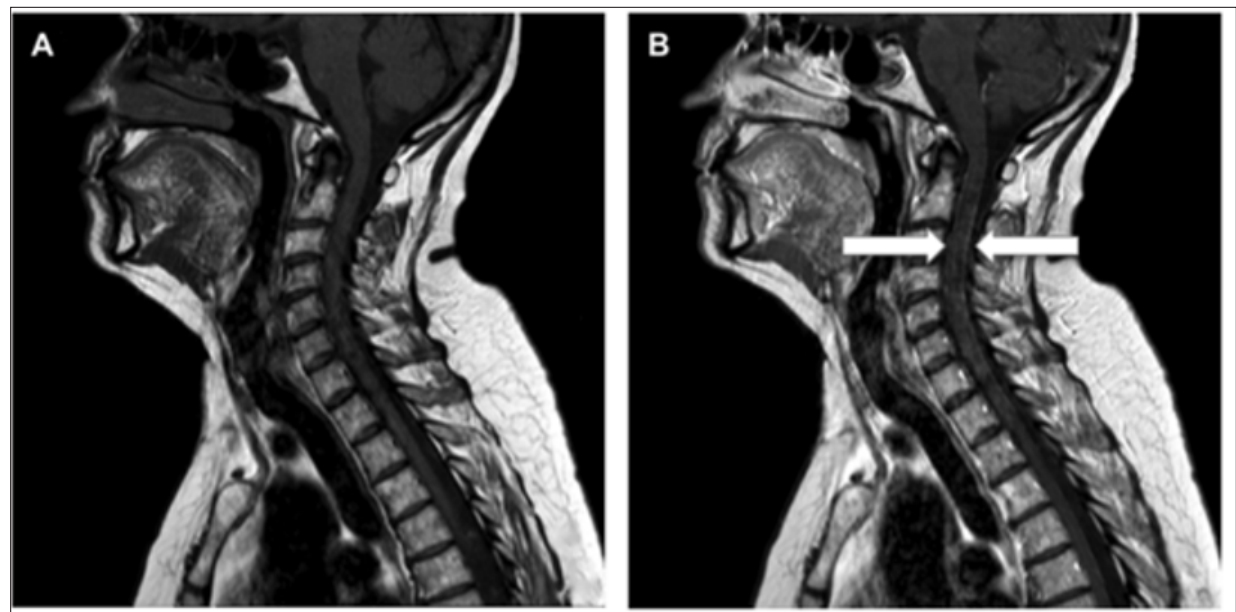

Figure 4: Spinal MRI.

a. The sagittal T1-weighted image of the upper spinal cord without gadolinium contrast;

b. T1-weighted image of the cervical spine with contrast enhancement along the dura, suggestive of leptomeningeal spread (white arrows).
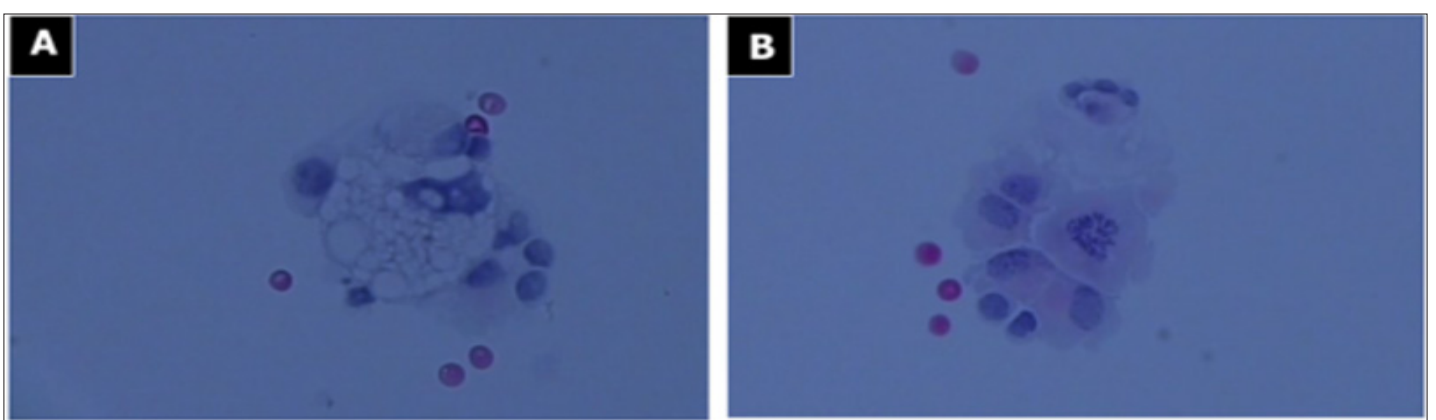

Figure 5: Histopathologic section of cerebrospinal fluid (CSF).

a. Atypical slightly degenerative giant glioblastoma cells (Papanicolaou, 400x);

b. CSF: vital atypical epithelioid glioblastoma cells (Papanicolaou, 400x). 
Table 1: Cerebrospinal fluid (CSF) analysis. The Patient had already received concomitant radiation and chemotherapy. Two months after surgery, the patient developed severe neck pain, became dizzy and was confused. The patient passed away four months after the initial diagnosis.

\begin{tabular}{|c|c|c|c|c|c|}
\hline Normal values (CSF) & Parameter & 1. day $0^{*}$ & 2. day $16^{*}$ & 3. day $22 *$ & 4. day $23^{*}$ \\
\hline & Appearance & clear & clear & clear & clear \\
\hline$-<1 / \mu \mathrm{l}$ & Erythrocytes & 31 & 5 & 16 & 14 \\
\hline$-<5 / \mu \mathrm{l}$ & Leukocytes & 1 & 8 & 1 & 1 \\
\hline $0.6-2.2 \mathrm{mmol} / \mathrm{l}$ & Lactate & 9.8 & 12.5 & 11.5 & 9.7 \\
\hline $15-45 \mathrm{mg} / \mathrm{dl}$ & Protein & 84 & 135 & 143 & 140 \\
\hline $40-70 \mathrm{mg} / \mathrm{dl}$ & Glucose & 10 & $<1$ & $<1$ & $<1$ \\
\hline
\end{tabular}

\section{Discussion}

It is well established that GBM can exhibit a range of morphological features. Rare presentations of granular cell GBM [810] and rhabdoid GBM [11-13] have been reported. However, these may also be mistaken for metastatic carcinoma. These rare forms of GBM frequently develop leptomeningeal dissemination and early recurrence, and the overall prognosis is considered extremely poor $[1,14]$. Kepes et al. were the first to report on E-GBM and provided a comprehensive description of adenoid GBM and GBM with epithelial metaplasia [15]. They described a pathognomonic tumor cell arrangement with cribriform, trabecular or pseudoacinar structures in five tumors. They were defined as adenoid structures in gliosarcoma $[1,15,16]$. The authors suggested that the pathological arrangement might help to distinguish the tumor from metastatic carcinomas, an interpretation in line with that of other authors [17]. Rubinstein et al. noted that these arrangements are rare GBM variants and described tumors with papillary formation mimicking medulloepithelial-like formations [18].

Concerning terminology, Kepes et al. defined them as adenoid tumors, but because of the variety of cell arrangements, the terminology has since shifted to the term epithelioid tumors [15]. Mork et al. reported on the papillary elements in adenoid GBM, which may mimic structures in medulloepithelioma [19] and on epithelial metaplasia in malignant gliomas [3]. E-GBMs represent one of the rarest morphological subtypes of glioblastoma [1]. Due to their neuroradiologic and morphologic features, they are often not suspected of being high-grade gliomas [20], and their well-circumscribed masses are often mistaken for metastatic carcinoma. As a rare variant of GBM, the epithelioid pattern may represent a very primitive tumor cell phenotype. Examples of malignant gliomas with lipidized tumor cells in which striking intracytoplasmic accumulations of lipid obscured the glial lineage of the lesion have been described [21-23]. Epithelioid neoplasms are defined as tumors with variable sized cells with eosinophilic cytoplasm, rounded cell morphology, nuclear atypia and mitotic figures [16]. Similarly, large eccentric nuclei and prominent nucleoli can occur but with no cytoplasmic ball-like filaments and no polyphenotypic immunohistochemical expression. The cells often display discohesion [23].
In addition, E-GBM might feature prominent xanthic changes and can be mistaken for metastatic renal cell carcinoma [1,21,24]. In 2008, Rodriguez defined epithelioid glioblastoma as round cells without a conspicuous process, with areas where the cells showed more eosinophilic cytoplasm and prominent nucleoli, as well as glial fibrillary acidic protein and CAM 5.2-immunoreactivity [1]. The radiological presentation of primary malignant epithelioid tumors of the central nervous system is non-specific. In MRI, the E-GBMs are iso- to hypointense on T1-weighted images, of mixed signal intensity on T2-weighted images and contrast-enhanced with gadolinium. As a pathognomonic feature, MRI findings without peripheral edema may be clinically significant [16], and newly diagnosed intracerebral masses without a recognized primary focus can be a sign of an epithelioid GBM [25]. In many cases, sharp circumscription and occasional hemorrhage are stronger clinical signs of metastatic tumor than primary glioma. Further signs include cystic, hemorrhagic, calcific and necrotic foci [20]. Epithelioid sarcoma is also a relatively rare lesion and more commonly seen in males.

In contrast to epithelioid sarcomas, epithelioid glioblastoma lesions are still characterized by their vascular proliferative changes and will stain with glial fibrillary acidic protein antibody (GFAP) [26]. Pediatric E-GBMs express the typical histological characteristics seen in adult tumors. Immunoreactivities for INI1 and loss of diverse protein expressions were seen in most cases [27], making the diagnosis of infiltrating astrocytomas difficult. Table 2 provides an overview of case reports on epithelioid glioblastoma and their immunohistochemistry. The majority of E-GBMs arise de novo. However, a small proportion of E-GBMs may progress from lower grade astrocytomas [28]. Alteration of BRAF V600E in particular has been reported as a possible driving mutation in the malignization of low-grade astrocytomas [29-33]. Exploration of molecular markers and targeted therapy has become the foundation and hope of current GBM research. Considering the rarity of E-GBMs, a delineation of BRAF V600E in large trials is highly unlikely. Nevertheless, its poor prognosis calls for extensive molecular characterization of possible target points in individual cases. 
Table 2: Reported cases of epithelioid glioblastoma (E-GBM) and their immunohistochemistry.

\begin{tabular}{|c|c|c|c|c|c|c|c|c|c|}
\hline Literature & $\begin{array}{l}\mathrm{N} \text { of } \\
\text { cases }\end{array}$ & GFAP & Vimentin & $\begin{array}{c}\text { INI-1 } \\
\text { (BAF47) }\end{array}$ & EMA & AE1/3 & CAM5.2 & Population & Age/Gender \\
\hline Akimoto et al. [16] & 1 & pos & pos & nd & nd & neg & nd & Adult & $43 / \mathrm{F}$ \\
\hline Rosenblum et al. [21] & 3 & pos & nd & nd & pos & pos & nd & Adult & 52/M; 60/F; 68/M \\
\hline $\begin{array}{c}\text { Kleinschmidt et al. } \\
{[7,20,23,34]}\end{array}$ & 16 & pos & nd & pos & neg & nd & nd & $\begin{array}{c}2 \text { pediatric/ } \\
14 \text { adult }\end{array}$ & $\begin{array}{c}\text { 10/F; 14/M; 18/M; } \\
\text { 18/M } \\
\text { 21/F; 24/M; 24/M; } \\
25 / \mathrm{M} \\
27 / \mathrm{F} ; 27 / \mathrm{F} ; 29 / \mathrm{M} ; \\
\text { 29/F } \\
\text { 41/M; 43/M; } \\
\text { 50/M; 69/M }\end{array}$ \\
\hline Rodriguez et al. [1] & 10 & pos & nd & pos & $\begin{array}{c}6 \text { pos } 3 \\
\text { neg }\end{array}$ & pos & neg & Adult & 44-63 5:5 (M:F) \\
\hline Gasco et al. [6] & 1 & pos & nd & nd & pos & pos & pos & Adult & $67 / \mathrm{M}$ \\
\hline Miyata et al. [35] & 2 & pos & nd & nd & pos & nd & nd & Adult & $\begin{array}{l}37 / \mathrm{M} \\
54 / \mathrm{F}\end{array}$ \\
\hline Tanaka et al. [25] & 1 & pos & nd & nd & nd & pos & nd & Adult & $55 / \mathrm{M}$ \\
\hline Broniscer et al. [27] & 6 & pos & nd & pos & neg & $\begin{array}{c}1 \text { pos } 5 \\
\text { neg }\end{array}$ & nd & Pediatric & $\begin{array}{c}3.5 / \mathrm{M} ; 3.5 / \mathrm{F} ; 5 / \mathrm{M} ; \\
10.7 / \mathrm{F} \\
10.2 / \mathrm{F} ; 11.3 / \mathrm{M}\end{array}$ \\
\hline Liebelt et al. [36] & 1 & pos & nd & nd & nd & nd & nd & Adult & $66 / \mathrm{W}$ \\
\hline Kuroda et al. [28] & 1 & pos & pos & pos & neg & nd & neg & Adult & $26 / \mathrm{F}$ \\
\hline Matsumura et al. [32] & 1 & pos & pos & pos & nd & nd & nd & Adult & $18 / \mathrm{M}$ \\
\hline Gill et al. [37] & 1 & pos & nd & nd & pos & nd & nd & Adult & $55 / \mathrm{M}$ \\
\hline Sugimoto et al. [38] & 4 & pos & pos & pos & neg & 3 pos & nd & $\begin{array}{l}1 \text { pediatric/ } \\
3 \text { adult }\end{array}$ & $\begin{array}{c}\text { 16/F; 19/F; 30/F; } \\
48 / \mathrm{F}\end{array}$ \\
\hline $\begin{array}{c}\text { Alexandrescu et al. } \\
{[29]}\end{array}$ & 11 & $\begin{array}{l}9 \text { pos } \\
2 \text { neg }\end{array}$ & $\begin{array}{l}5 \text { pos } \\
6 \text { nd }\end{array}$ & $\begin{array}{l}4 \text { pos } \\
7 \text { nd }\end{array}$ & $\begin{array}{l}8 \text { pos } \\
3 \text { neg }\end{array}$ & nd & nd & $\begin{array}{l}5 \text { pediatric/ } \\
6 \text { adult }\end{array}$ & $\begin{array}{l}\text { 2/M; 11/M; 11/M; } \\
\text { 12/M 14/M; 21/F; } \\
\text { 26/M; 38/M } \\
\text { 56/M; 70/F; 79/M; }\end{array}$ \\
\hline
\end{tabular}

Note: EMA, Epithelial membrane antigen; F, female; GFAP, Glial fibrillary acid protein; M, male; pos, positive; neg, negative; nd, not done

While the status of this marker was not determined in this patient, targeted pharmacological agents such as the BRAF enzyme inhibitor Vemurafenib may represent an additional option in the treatment of E-GBM in the future [34]. In summary, the current case and the literature review report the pathologic, immunophenotypic and molecular characteristics of epithelioid GBMs. Although molecular abnormalities of E-GBMs overlap with those of typical GBMs and gliosarcomas, they are a specific subgroup of GBMs that must be considered when metastatic carcinomas are suspected. To our knowledge, we also provide the first report about an E-GBM with leptomeningeal dissemination. This may be of importance since E-GBMs are associated with an even worse prognosis compared with other, more frequent GBM-types.

\section{Conclusion}

E-GBM is histologically characterized by the presence of epithelial-like cells. Because of their inconspicuous appearance to surgeons and on MRI scans, E-GBMs are often not recognized as high-grade gliomas, highlighting the importance for clinicians to be aware of their unusual patterns. Therefore, E-GBM should be considered as a differential diagnosis even when a known primary systemic malignancy is present because its histopathological features may mimic those of metastatic brain adenocarcinomas.

\section{Acknowledgment}

We thank Prof. Dr. W. Paulus (Department of Neuropathology, University Clinic Muenster, Germany) for his pathology expertise.

\section{Patient consent}

The patient is deceased; the legal supervisor has consented.

\section{References}

1. Rodriguez FJ, Scheithauer BW, Giannini C, Bryant SC, Jenkins RB (2008) Epithelial and pseudoepithelial differentiation in glioblastoma and gliosarcoma: a comparative morphologic and molecular genetic study. Cancer 113(10): 2779-2789.

2. Louis DN, Ohgaki H, Wiestler OD, Cavenee WK, Burger PC, et al. (2007) The 2007 WHO classification of tumours of the central nervous system. Acta Neuropathol 114(2): 97-109. 
3. Mork SJ, Rubinstein LJ, Kepes JJ, Perentes E, Uphoff DF (1988) Patterns of epithelial metaplasia in malignant gliomas. II. Squamous differentiation of epithelial-like formations in gliosarcomas and glioblastomas. J Neuropathol Exp Neurol 47(2): 101-118.

4. Du Plessis DG, Rutherfoord GS, Joyce KA, Walker C (2004) Phenotypic and genotypic characterization of glioblastoma multiforme with epithelial differentiation and adenoid formations. Clin Neuropathol 23(4): 141-148.

5. Ozolek JA, Finkelstein SD, Couce ME (2004) Gliosarcoma with epithelial differentiation: immunohistochemical and molecular characterization. A case report and review of the literature. Mod Pathol 17(6): 739-745.

6. Gasco J, Franklin B, Fuller GN, Salinas P, Prabhu S (2009) Multifocal epithelioid glioblastoma mimicking cerebral metastasis: case report. Neurocirugia (Astur) 20(6): 550-554.

7. Kleinschmidt De Masters BK, Aisner DL, Birks DK, Foreman NK (2013) Epithelioid GBMs show a high percentage of BRAF V600E mutation. Am J Surg Pathol 37(5): 685-698.

8. George AA, Fuller GN, Langford LA, Simon CD, Zingalis AA, et al. (2013) Unusual presentation of a granular cell astrocytoma. Histopathology 63(6): 883-885.

9. Kim KH, Song JY, Choi CH, Kim L, Choi SJ, et al. (2012) Granular cell astrocytoma: report of a case. Korean J Pathol 46(4): 370-372.

10. Yao K, Wang H, Duan Z, Bian Y, Xia L, et al. (2014) Mixed granular cell astrocytoma and fibrosarcoma of the brain: a case report. Int J Clin Exp Pathol 7(7): 4473-4478.

11. Babu R, Hatef J, Mc Lendon RE, Cummings TJ, Sampson JH, et al. (2013) Clinicopathological characteristics and treatment of rhabdoid glioblastoma. J Neurosurg 119(2): 412-419.

12. Byeon SJ, Cho HJ, Baek HW, Park CK, Choi SH, et al. (2014) Rhabdoid glioblastoma is distinguishable from classical glioblastoma by cytogenetics and molecular genetics. Hum Pathol 45(3): 611-620.

13. Chen SC, Lin DS, Lee CC, Hung SC, Chen YW, et al. (2013) Rhabdoid glioblastoma: a recently recognized subtype of glioblastoma. Acta Neurochir (Wien) 155(8): 1443-1448; discussion 1448.

14. Momota H, Iwami K, Fujii M, Motomura K, Natsume A, et al. (2011) Rhabdoid glioblastoma in a child: case report and literature review. Brain Tumor Pathol 28(1): 65-70.

15. Kepes JJ, Fulling KH, Garcia JH (1982) The clinical significance of "adenoid" formations of neoplastic astrocytes, imitating metastatic carcinoma, in gliosarcomas. A review of five cases. Clin Neuropathol 1(4): 139-150.

16. Akimoto J, Namatame H, Haraoka J, Kudo M (2005) Epithelioid glioblastoma: a case report. Brain Tumor Pathol 22(1): 21-27.

17. Galloway PG, Roessmann U (1986) Anaplastic astrocytoma mimicking metastatic carcinoma. Am J Surg Pathol 10(10): 728-732.

18. Rubinstein LJ, Mork SJ, Kepes JJ, Uphoff DF (1986) Squamous Differentiation of Epithelial-Like Formations in Glioblastoma and Gliosarcoma. Journal of Neuropathology and Experimental Neurology 45: 327-327.

19. Mork SJ, Rubinstein LJ, Kepes JJ (1988) Patterns of epithelial metaplasia in malignant gliomas. I. Papillary formations mimicking medulloepithelioma. J Neuropathol Exp Neurol 47(2): 93-100.

20. Kleinschmidt De Masters BK, Alassiri AH, Birks DK, Newell KL, Moore W, et al. (2010) Epithelioid versus rhabdoid glioblastomas are distinguished by monosomy 22 and immunohistochemical expression of INI-1 but not claudin 6. Am J Surg Pathol 34(3): 341-354.

21. Rosenblum MK, Erlandson RA, Budzilovich GN (1991) The lipid-rich epithelioid glioblastoma. Am J Surg Pathol 15(10): 925-934
22. Kepes JJ, Rubinstein LJ (1981) Malignant gliomas with heavily lipidized (foamy) tumor cells: a report of three cases with immunoperoxidase study. Cancer 47(10): 2451-2459.

23. Kleinschmidt De Masters BK, Meltesen L, Mc Gavran L, Lillehei KO (2006) Characterization of glioblastomas in young adults. Brain Pathol 16(4): 273-286

24. Tanaka S, Nakada M, Nobusawa S, Suzuki SO, Sabit H, et al. (2014) Epithelioid glioblastoma arising from pleomorphic xanthoastrocytoma with the BRAF V600E mutation. Brain Tumor Pathol 31(3): 172-176.

25. Tanaka S, Nakada M, Hayashi Y, Nakada S, Sawada Kitamura S, et al. (2011) Epithelioid glioblastoma changed to typical glioblastoma: the methylation status of MGMT promoter and 5-ALA fluorescence. Brain Tumor Patholc 28(1): 59-64.

26. Prayson RA, Chahlavi A (2002) Metastatic epithelioid sarcoma to the brain: palisaded necrosis mimicking glioblastoma multiforme. Ann Diagn Pathol 6(5): 302-306.

27. Broniscer A, Tatevossian RG, Sabin ND, Klimo P, Dalton J, et al. (2013) Clinical, radiological, histological, and molecular characteristics of paediatric epithelioid glioblastoma. Neuropathol Appl Neurobiol 40(3): 327-336.

28. Kuroda J, Nobusawa S, Nakamura H, Yokoo H, Ueda R, et al. (2016) A case of an epithelioid glioblastoma with the BRAF V600E mutation colocalized with BRAF intact low-grade diffuse astrocytoma. Neuropathology 36(2): 181-186.

29. Alexandrescu S, Korshunov A, Lai SH, Dabiri S, Patil S, et al. (2016) Epithelioid Glioblastomas and Anaplastic Epithelioid Pleomorphic Xanthoastrocytomas-Same Entity or First Cousins? Brain Pathol 26(2): 215-223.

30. Takahashi Y, Akahane T, Sawada T, Ikeda H, Tempaku A, et al. (2015) Adult classical glioblastoma with a BRAF V600E mutation. World J Surg Oncol 13: 100 .

31. Suzuki Y, Takahashi Fujigasaki J, Akasaki Y, Matsushima S, Mori R, et al. (2016) BRAF V600E-mutated diffuse glioma in an adult patient: a case report and review. Brain Tumor Pathol 33(1): 40-49.

32. Matsumura N, Nakajima N, Yamazaki T, Nagano T, Kagoshima K, et al. (2017) Concurrent TERT promoter and BRAF V600E mutation in epithelioid glioblastoma and concomitant low-grade astrocytoma. Neuropathology 37(1): 58-63.

33. Funata N, Nobusawa S, Yamada R, Shinoura N (2016) A case of osteoclastlike giant cell-rich epithelioid glioblastoma with BRAF V600E mutation. Brain Tumor Pathol 33(1): 57-62.

34. Kleinschmidt De Masters BK, Aisner DL, Foreman NK (2015) BRAF VE1 immunoreactivity patterns in epithelioid glioblastomas positive for BRAF V600E mutation. Am J Surg Pathol 39(4): 528-540.

35. Miyata H, Ryufuku M, Kubota Y, Ochiai T, Niimura K, et al. (2012) Adultonset angiocentric glioma of epithelioid cell-predominant type of the mesial temporal lobe suggestive of a rare but distinct clinicopathological subset within a spectrum of angiocentric cortical ependymal tumors. Neuropathology 32(5): 479-491.

36. Liebelt BD, Boghani Z, Takei H, Fung SH, Britz GW (2015) Epithelioid glioblastoma presenting as massive intracerebral hemorrhage: Case report and review of the literature. Surg Neurol Int 6(2): S97-S100.

37. Gill SK, Padmanabhan V, Hickey WF, Marotti JD (2015) Glioblastoma multiforme with epithelial differentiation: a potential diagnostic pitfall in cerebrospinal fluid cytology. Diagn Cytopathol 43(8): 638-641.

38. Sugimoto K, Ideguchi M, Kimura T, Kajiwara K, Imoto H, et al. (2016) Epithelioid/rhabdoid glioblastoma: a highly aggressive subtype of glioblastoma. Brain Tumor Pathol 33(2): 137-146. 
ISSN: 2574-1241

DOI: $10.26717 / B J S T R .2018 .10 .001920$

Walid Albanna. Biomed J Sci \& Tech Res

CC (i) This work is licensed under Creative

Submission Link: https://biomedres.us/submit-manuscript.php

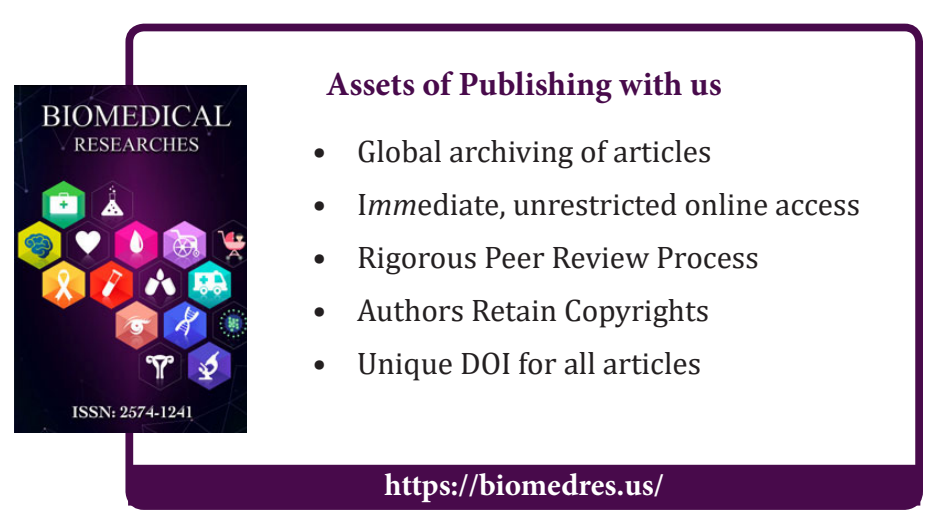

